\title{
La obra concretista de Stanisław Dróżdż. Evolución y recepción
}

\author{
Marcin KUREK \\ Uniwersytet Wrocławski (Polonia) \\ jusmar@interia.pl
}

Recibido: Mayo de 2012

Aceptado: Diciembre de 2012

\section{Resumen}

Stanisław Dróżdż (1939-2009), el máximo representante de la poesía concreta y visual en Polonia, evolucionó en su obra desde la poesía literaria hasta la pictórica de gran tamaño, tridimensional y performativa. La problemática de la recepción de su obra está estrechamente relacionada con esta evolución.

Palabras clave: Stanisław Dróżdż, poesía polaca, poesía concreta, poesía visual

The concrete poetry of Stanislaw Dróżdż. Evolution and reception.

\begin{abstract}
Stanisław Dróżdż (1939-2009), the most important representative of concrete and visual poetry in Poland, evolved in his work from the literary poetry to the pictorial one, three-dimensional and performative. The problem of the reception of his work is closely related to this evolution.
\end{abstract}

Key words: Stanisław Dróżdż, Polish poetry, concrete poetry, visual poetry

La obra poética de Stanisław Dróżdż (1939-2009), uno de los personajes-clave de la poesía experimental y visual contemporánea de Polonia, se sitúa en un espacio heterogéneo entre el concretismo literario y el arte conceptual -el propio artista, desde el primer artículo-manifiesto de 1968, nombraba a todas sus obras como pojęcioksztalty (concepto-formas) (DRÓŻDŻ 1968: 73-76) - por lo cual toda problemática relacionada con su recepción está centrada en torno a una serie de cuestiones principales que comentaremos en el presente artículo. 
Se trata de un arte vanguardista - una poesía no literaria y una obra pictográfica no figurativa- planteado y practicado en el ámbito político e ideológico de un país bajo el régimen comunista, al menos en su primera fase hasta el año 1989. Siendo una obra novedosa y radicalmente experimental en sí, afronta la natural dificultad de recepción tanto por parte de los lectores y/o expectadores como por parte de la crítica profesional, especialmente la literaria. Es un autor minoritario, durante casi toda la carrera situado en las afueras de cualquier mainstream institucional, apoyado por un grupo de amigos artistas, críticos de arte, jefes de galerías, en déficit de recursos propios, y también tecnológicos, para preparar y exponer algunas de sus obras.

La recepción de la poesía -también de la poesía visual y dentro de ella de la poesía concreta- desde finales de los años 60 ha sido determinada en Polonia no solamente por su problemática de género sino también por las circunstancias políticas e históricas. El movimiento concretista polaco, convergente con las experiencias occidentales desarrolladas básicamente en los años 50 y 60, en caso de Stanisław Dróżdż es efecto de un proceso intelectual y creativo individual e original (inspirado en los experimentos de la vanguardia polaca de entreguerras) cuyo punto de partida es una poesía literaria en su variante lingüística. No es de prescindir el que Dróżdż inicie su trayectoria artística como poeta literario en Wrocław, capital de Baja Silesia, la ciudad que a partir de la década de los 60 se convierte en un innegable punto de referencia en el mapa cultural del país con el Teatro Laboratorium de Jerzy Grotowski (desde 1965), la revista mensual Odra (desde 1961) y con las figuras emblemáticas de la literatura de posguerra como Tadeusz Różewicz (desde 1968), Urszula Kozioł (desde 1958), Rafał Wojaczek (desde 1965) o Tymoteusz Karpowicz (hasta 1973), el máximo representante y promotor de la poesía lingüística polaca. Veamos algunos poemas tradicionales de Dróżdż.

En mayo de 1969 la revista Odra (DRÓŻDŻ 1969: 79-80) publica ocho poemas breves de los que citaremos tres:

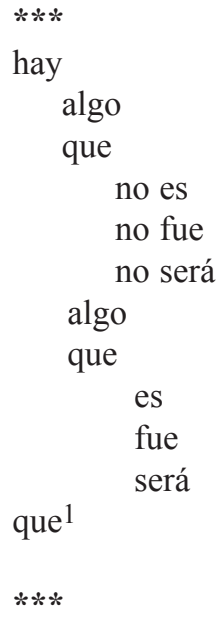

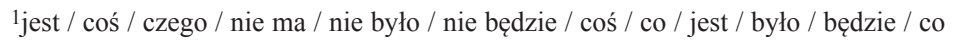




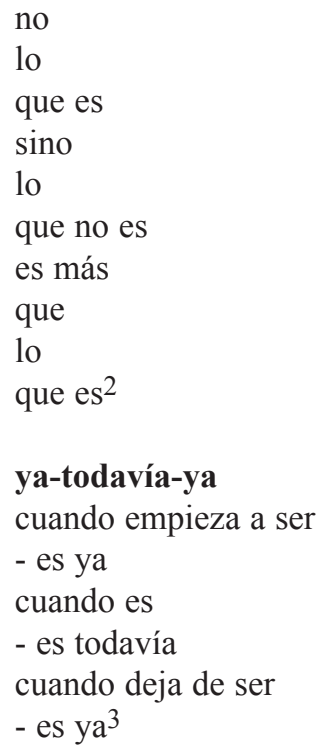

Los textos citados, perterencientes a un ciclo titulado Las miniaturas, publicados en una revista es decir el canal de distribución más tradicional, son poemas conceptuales, minimalistas, filosóficamente centrados en cuestiones ontológicas primarias. Al mismo tiempo su sintetistmo formal e ingenio lingüístico ya anuncian el paso decisivo hacia la poesía concreta, interesada en la palabra en sí. Ya desde aquel momento Dróżdż define el conjunto relativamente limitado de vocablos que posteriormente utilizará en sus obras concretistas más relvantes (ŁUBOWICZ 2009: 21). Más tarde veremos cómo la idea del primer poema -el juego con las formas temporales del verbo "ser"- evoluciona en una serie de rótulos permutatorios y cómo se aprovecha el espacio público urbano para su divulgación. También la base bicolor, blanco y negro de un texto impreso, se va a mantener en toda su obra.

Al hablar del contexto ideológico de la poesía de posguerra hay que considerar dos fenómenos. Por un lado el interés de las autoridades nacionales y regionales de la ciudad de Wroclaw y de Baja Silesia, una zona que apenas hacía 20 años entraba a formar parte de la República Popular de Polonia (hasta 1945 en Alemania), en promover todo tipo de actividades culturales, sobre todo entre los jóvenes, como muestra o justificación de la legitimidad de la administración polaca sobre estos terrenos (la frontera no reconocida por la RFA hasta 1970). Por otro lado, a mediados de los 60 , con la madurez de la primera generación de postguerra, se hace patente la crisis general del idioma oficial del estado, de la propaganda, omnipresente en la prensa, la radio y también en gran parte de la literatura oficial (la no oficial no existe hasta mediados de los 70 cuando nace samizdat polaco). Esa crisis general del idioma atascado entre la neolengua peculiar totalitaria y el mundo no representado de la lite-

2 nie / to / co jest / a / to / czego nie ma / jest bardziej / niż / to / co jest

3 już - jeszcze - już: gdy być zaczyna / - to już / gdy jest / - to jeszcze / gdy być przestaje / - to już 
ratura controlada por la censura, desemboca en una serie de fenómenos poéticos como el grupo Nowa Fala (Nueva Ola) encabezado, entre otros, por Adam Zagajewski, poeta hoy en día comúnmente conocido en España, con su temprana poética de lingüísmo entendido como crítica de la lengua oficial (KORNHAUSER 1974, GŁOWIŃSKI 2009), o el movimiento concretista, fundado y presidido por Dróżdż y su cuestionamiento general de la eficacia del sistema y de la narratividad como portadora de significados. En resumidas cuentas: los jovenes critican la lengua directamente o la esquivan.

Si la evolución que observamos en la poesía concreta es desde el idioma hacia la imagen -decía Dróżdż: "Las palabras no mienten y las frases mienten. Una palabra no puede mentir. Y cuando se juntan tres palabras ya empieza la mentira" (ŁUBOWICZ 2009: 26)- al mismo tiempo la tendencia entre los artistas es de abandonar la imagen a favor de la idea. El conceptualismo aparece como alternativa para el arte figurativo realista, preferido y promovido por las autoridades (un decreto del partido comunista polaco de 1960 limitaba a un $15 \%$ el volumen de cuadros abstractos permitidos en cada exposición). En ambos casos (concretismo y conceptualismo) la palabra queda aislada de su contexto narrativo, se convierte en un objeto, es privada de su funcionalidad, lo cual permite evitar las trampas del idioma de la propaganda y del arte a servicios de la política. En este punto hay que subrayar, no obstante, que la obra de Dróżdż en todas sus fases, conforme con sus declaraciones hechas durante las conversaciones con Dawidek Gryglicka (DAWIDEK 2012: 92), está libre de cualquier intención política. Diríamos en este caso que el contexto político se le impone de fuera, siendo que en una situación de la dictadura política nada se libera de un contexto político.

Junto con el proceso de conceptualización, las obras del autor polaco adquieren carácter cada vez más plástico, tridimensional y exposicional, lo cual redirige la recepción crítica desde el enfoque literario, bastante desarmado ante el fenómeno, hasta la corriente principal de la crítica de arte que absorbe la obra de Dróżdż como uno de los ejemplos más interesantes del arte conceptual polaco. También la situación del concretismo en una zona fronteriza entre la literatura y el arte permite cruzar, fundir dos ámbitos artísticos, el de poetas y el de pintores, y ampliar notablemente el alcance de la posible recepción particular y profesional. De hecho, desde el primer momento la poesía concreta se difunde en libros, catálogos y revistas pero también se exhibe en galerías, clubes universitarios, bibliotecas, teatros, museos (desde el primer estreno en febrero de 1968, en Wroclaw y en su provincia, tienen lugar tres primeras presentaciones nacionales de la poesía concreta): todo ello en forma de exposición pictórica, donde los poemas concebidos por Dróżdż son convertidos en rótulos y expuestos como cuadros. Consciente de su dificultad interpretativa, el artista invita a jóvenes críticos y teóricos de arte y de literatura a participar en las presentaciones concretistas de modo que la poesía casi siempre viene acompañada de interpretaciones y comentarios competentes y favorables (por ej. de Grzegorz Dziamski o Tadeusz Sławek, hoy catedráticos de arte y de literatura comparada), a diferencia de las críticas en la prensa (DAWIDEK 2012: 86).

Con todo eso el jóven arte polaco se universaliza: separado de sus coetáneos por el telón de hierro participa en una corriente mundial (concretismo, conceptualismo, 
mail art) pero mantiene su carácter y contexto propios (abandono de la lengua como herramienta de falseamiento). Antes de que el movimiento concretista occidental pase a su fase de declive, Dróżdż logra participar como único representante de Polonia en la famosa exposición ambulante sound text - concrete poetry - visual texts de 1970-72 (Holanda, Alemania, Inglaterra), que recapitula los logros del movimiento concretista mundial. Y como muestra del problema de la recepción y aceptación del concretismo por parte de los círculos literarios, se puede mencionar que en los años 70 la solicitud de admisión que Dróżdż presentó a la Unión de Escritores Polacos, dirigida en aquel entonces por el poeta y novelista Jarosław Iwaszkiewicz, fue rechazada con el argumento de que la cartera de obras presentada "no fue un libro" (DAWIDEK 2012: 95).

En el contexto internacional de la recepción, interesa decir que a pesar del próposito concretista de suprimir la lengua como herramienta de la narración y de la política, Dróżdż sostuvo que sólo algunas de sus obras eran susceptibles de la traducción y sólo éstas se exhibían en las exposiciones internacionales. Como ejemplo citemos la famosa między (entre), estrenada en la Galería Foksal de Varsovia en 1977 y posteriormente exhibida en diferentes países. Se trata de un espacio cúbico blanco de unos $20 \mathrm{~m} 2$, cerrado pero transitable, con letras que componen la palabra "entre" dispersas en todas las paredes, el techo y el suelo, en diferentes sentidos, siendo que en ningún momento aparezca la palabra entrera. Los visitantes entran y salen por dos cortinas integradas en las paredes. En sus presentaciones internacionales la obra siempre mantenía la misma disposición de letras polacas, traduciéndose exclusivamente el título a la entrada. Como declaraba el autor: "La poesía concreta en mis realizaciones debe ser acontextual. Es que no está adscrita ni al lugar, ni al tiempo. Ha sido exhibida en China y Japón, en Austria y Australia y si sólo es posible traducir el título Entre a otra lengua, cada uno que esté dentro está en su casa" (DAWIDEK 2012: 88). La traducción de la palabra que compone la obra supondría un abuso: "No lo admito. El trabajo está acabado. No se multiplica, no se autofalsifica" (DAWIDEK 2012: 90). La palabra "entre" mantenida siempre en polaco refuerza la independencia de la obra de arte y la sensación de que, aunque literalmente entremos dentro de un poema, éste mantendrá su autonomía. Para la crítica polaca es una obra que revoluciona el modo de funcionamiento de un texto en el espacio (DAWIDEK 2010: 227) o que sitúa al lector entre las letras colocándolo en la situación de una mosca pisando un libro o un periódico (SŁAWEK 1989).

En su evolución hacia la plasticidad cada vez más radical y luego hacia la tridimensionalidad (ŁUBOWICZ 2009: 23), queda más bien claro que solamente los poemas depurados de cualquier huella de signo lingüístico, aunque sea una letra, es decir las obras conceptuales, poemas-objeto o instalaciones, son las que consiguen un grado deseado de universalidad. Se trata especialmente de dos obras excepcionales: la primera, significativamente llamada Poesía concreta (con el subtítulo Hilos), exhibida por pimera vez en la galería Foksal de Varsovia en 2002, consiste en colocar dentro de un cuarto 10 kilómetros de hilo de pescar creando así un espacio cúbico no transitable, que se contempla desde fuera (por las puertas), adecuadamente iluminado con una luz suave, sin que se vean los ganchos y puntos de amarre del hilo. Al mirar hacia dentro, creemos ver el fragmento de un espacio interminable, como 
imagen de algo misterioso, denso, primigenio. Algo inexpresable mediante cualquier otro código, sobre todo la lengua. Igual que las conocidas series de rótulos con preposiciones (antes-después, delante-detrás), otro signo identificador del artista, Poesía concreta. Hilos aborda el problema de lo inexpresable, susceptible de marcarse, definirse mediante los bordes pero no de nombrarse de forma directa. En una de las coversaciones Dróżdż declaraba: "he decidido crear algo que no tenga principio ni fin, algo que haga volar los marcos del inicio y del fin. Y espero que en gran medida lo haya conseguido. No toda poesía concreta aprovecha este elemento, ha sido un punto neurálgico para mí. [...] Los Hilos han sido montados, insertados en el suelo y en el techo y en las paredes, de forma que el hilo salía de alguna parte, desde la luna, atravesaba la galería, solamente la cruzaba, y nosotros contemplábamos exactamente este fragmento. No había principio ni fin" (DAWIDEK 2012: 88-89). La obra tiene también su significado autotélico siendo que los Hilos se estrenó 25 años después de entre, exactamente en la misma galería y en la misma sala lo cual permite interpretar la segunda obra en el sentido intertextual, como si la red de hilos representara todas las posibles conexiones de letras de la obra anterior (DAWIDEK 2010: 233).

La segunda obra, Alea iacta est, de carácter performativo y aletórico por excelencia, fue preparada como propuesta para representar a Polonia en la Bienal de Venecia en el año 2003. En medio del pabellón se colocó una mesa tapizada con tela verde con un juego de 6 dados y en las paredes que la rodeaban se colocaron casi 300.000 dados que mostraban todos los resultados posibles de un golpe de 6 piezas que estaban en la mesa. Los expectadores eran invitados (por los altavoces en 40 idiomas) al juego que consistía en tirar los dados y buscar en la pared el resultado correspondiente: "eres partícipe del juego / tira los dados / ordénalos en fila / encuentra el resutado en la pared / entre las 46656 posibles combinaciones / si encuentras, has ganado / si no, has perdido" (DAWIDEK 2010: 255). Dada la extrema dificultad de la búsqueda, la inmensa mayoría de los jugadores pierde. Las posibles interpretaciones van desde el azar en la vida humana, hasta la impotencia cognitiva. Una visitante argentina nada más entrar le preguntó a Dróżdż si la obra tenía que ver algo con Dios (DAWIDEK 2012: 204).

El problema de la recepción está también relacionado con el desfase que se produjo entre la concepción de muchas de las obras del autor y la posibilidad técnica de su realización. Respecto a esta cuestión, la obra de Dróżdż coincide con una característica muy importante del arte contemporáneo: la no existencia o el menosprecio del original y no la ejecución personal de la obra por el artista (ŁUBOWICZ 2009: 30). Además, Dróżdż quien desde el nacimiento padecía una enfermedad ósea que a partir de sus 40 años de edad lo tuvo atado a una silla de ruedas, tenía que recurrir a la ayuda física de sus amigos artistas para ejecutar los rótulos o cuadros con sus poemas. Posteriormente lo realizaban las galerías y museos por su cuenta, según el borrador presentado por el poeta. También en algunos casos para ejecutar una obra ideada, concebida con papel y lápiz, había que esperar décadas hasta que aparecieran ordenadores personales y sus procesadores de texto que permitían poner la idea en práctica. Este fue el caso de los cálculos necesarios para Alea iacta est o de las series de poemas titulados Textos numéricos, concebidos a principios de los $70 \mathrm{y}$ exhibidos en la forma deseada por el artista veinte años más tarde: en cada cuadro 
uno de los números desaparece porque está formado por números iguales que los del fondo. Las primeras versiones de los poemas, hechas con máquina de escribir, no garantizaban el efecto deseado.

Otra dimensión de la poesía visual que abre nuevas posibilidades cara a la recepción es su potencial presencia en el espacio público. En 1998, una agencia publicitaria de Poznań (AMS) inició su proyecto titulado "Galería exterior" que consistía en exhibir en 400 carteles publicitarios en todo el país una obra plástica de algún artista nacional. En 2001 se exhibió la obra de Dróżdż titulada Bez tytułu (lub) (Sin título [o]), desconcertando a los transeuntes, convencidos de que se trataba de una campaña publicitaria que iba a subrayar las ventajas de un producto sobre otro. Se trataba de una superficie blanca con preposición lub (o) repetida varias veces. Dos años más tarde la misma obra fue exhibida en la fachada del Centro Cultural de Alta Silesia en Katowice. Lo que se demostraba aquí era que el arte podía literalmente salir del museo a la calle, además la falta del marco y la forma curvada del edificio reforzaban la sensación de una obra abierta que trascendía todos los límites de la recepción institucional a través del sistema tipo. Lo interesante era ver cómo el mismo poema concreto, al pasar de un cartel a la fachada de un museo, perdía su connotación comercial.

Otro ejemplo de la exposición de un poema concreto en el espacio urbano es el más reciente y viene de Wrocław: el poema literario citado ("algo / que / es / fue / será") encuentra su desarrollo en un poema concreto titulado Klepsydra (Reloj de arena). La obra en su versión básica fue concebida en 1967 como rótulo con las tres palabras "SERÁ-ES-FUE" colocadas verticalmente, de las que la primera y la última, multiplicadas, en el extremo superior e inferior mantenían el tamaño máximo, reduciéndose hacia la palabra central "ES", la más pequeña. Posteriormente, la idea fue desarrollada en una serie permutatoria de 54 rótulos que agotaban todas las combinaciones de las tres palabras. Las palabras venían intercaladas, unas mayores que otras, en función de la importancia que le diéramos a cada una de las perspectivas temporales. En el primer cuadro lo que adquiere mayor importancia es el futuro más lejano y el pasado más remoto mientras que el presente es puntual y casi desapercibido, lo que corresponde a la forma de percepción típica del tiempo humano. La reproducción permanente del primer poema de la serie acaba de ser exibida en la fachada del Museo Contemporáneo de Wroclaw situado en un búnquer alemán de la segunda guerra mundial. Aquí también la obra se sale del museo, como en el caso anterior pero, desde el punto de vista de la posible recepción, lo importante es que el búnquer está situado en una de las avenidas principales de la ciudad y el poema puede ser contemplado diariamente por varios miles de personas. Además el poema de un autor relacionado con Wroclaw se convierte en un emblema del museo y de la ciudad.

Guillermo de Torre decía con su lucidez premonitaria que el porvenir del concretismo "-no diremos exactamente literario pero, sí visual- está ligado muy directamente con la evolución de la plástica, del diseño industrial, de la publicidad, del cinematógrafo, de todas aquellas formas ya inventadas o por inventar en que la imagen y el signo se adelantan sobre la palabra" (TORRE DE 1965: 760). A partir de los ejemplos comentados queremos creer que la historia del concretismo y de sus oportunidades no está acabada. 


\section{Referencias bibliográficas}

DAWIDEK GRYGLICKA, M. (2010): „Międzysłowia. O twórczości Stanisława Dróżdża. Interpretacje”, Dyskurs, 10/2010, pp. 218-258.

DAWIDEK GRYGLICKA, M. (2012): Odprysk poezji. Stanisław Dróżż mówi, Korporacja Ha!Art-Narodowe Centrum Kultury, Kraków-Warszawa.

DRÓŻDŻ, S. (1968): "Pojęciokształty”, Odra, 4/1968, pp. 74-76.

DRÓŻDŻ S. (1969): “Miniatury”, Odra, 5/1969, p. 79-80.

GŁOWIŃSKI, M. (2009): Nowomowa i ciagi dalsze. Szkice dawne i nowe, Universitas, Kraków.

KORNHAUSER, J., ZAGAJEWSKI, A., (1974): Świat nie przedstawiony, Wydawnictwo Literackie, Kraków.

ŁUBOWICZ, E. (2009): "Rzeczywistość jest tekstem. O «pojęciokształtach» Stanisława Dróżdża" en ŁUBOWICZ, E. (red.), Stanisław Dróżdż. Poczq̨tekoniec. Pojęcioksztalty. Poezja konkretna, Ośrodek Kultury i Sztuki, Wrocław, pp. 19-35.

SŁAWEK, T. (1989): Między literami, Wydawnictwo Dolnośląskie, Wrocław.

TORRE DE, G. (1965): Historia de las literaturas de vanguardia, Visor Libros, Madrid. 